\title{
DC Stark addressing for quantum memory in Tm:YAG
}

\author{
Konstantin Gerasimov ${ }^{1}$, Mansur Minnegaliev ${ }^{1}$, Ravil Urmancheev ${ }^{1, *}$, and Sergey Moiseev ${ }^{1}$ \\ ${ }^{1}$ Kazan Quantum Center, Kazan National Research Technical University named after A.N. Tupolev - \\ KAI, Kazan, 420111 Russia
}

\begin{abstract}
We observed a linear DC Stark effect for ${ }^{3} \mathrm{H}_{6}-{ }^{3} \mathrm{H}_{4}$ optical transition of $\mathrm{Tm}^{3+}$ ions in $\mathrm{Y}_{3} \mathrm{Al}_{5} \mathrm{O}_{12}$. We observed that application of electric field pulse suppresses the two-pulse photon echo signal. If we then apply a second electric pulse of opposite polarity the echo signal is restored again, which indicates the linear nature of the observed effect. The effect is present despite the $\mathrm{D}_{2}$ symmetry of the $\mathrm{Tm}^{3+}$ sites that prohibits a linear Stark effect. Experimental data analysis shows that the observed electric field influence can be attributed to defects that break the local crystal field symmetry near $\mathrm{Tm}^{3+}$ ions. Using this effect we demonstrate selective retrieval of light pulses in two-pulse photon echo.
\end{abstract}

The development of optical quantum technologies such as quantum computer and longdistance quantum communications stimulates the creation of quantum memories, devices that would broaden their possible applications. One of the promising media in the field of quantum memories is the multi-atomic ensembles that allow one to strengthen the interaction with weak photon fields. Rare-earth ions stand out among such systems due to remarkable combination of long $\mathrm{T}_{2}$ coherence times and broad inhomogeneous optical transitions and possibility to realize a long-time storage on electron-nuclear spin sub-levels.

$\mathrm{Tm}^{3+}:$ YAG crystal has been extensively investigated due to its laser applications and its $793 \mathrm{~nm}$ wavelength transition ${ }^{3} \mathrm{H}_{6}-{ }^{3} \mathrm{H}_{4}$ that lies in the third optical fibre transparency window. Tm ions in this material substitute $\mathrm{Y}$ ions in crystallographic sites with $\mathrm{D}_{2}$ local symmetry. Linear Stark effect is absent in central symmetries [1] such as $\mathrm{D}_{2}$. However in this work we demonstrate a quasi-linear Stark effect during the two-pulse photon echo formation at $4 \mathrm{~K}$ temperature.

In the experiments wave vectors of the incoming pulses were oriented along the [1 $\overline{1} 0]$ crystallographic axis. Both pulses were polarised along the [001] axis. In these conditions all exited thulium atoms had the same Rabi frequency. We observed the suppression of the photon echo after the applied electric field pulse. The echo was suppressed completely after the pulse with $\geq 5 \mu$ s duration and $E \geq 500 \mathrm{~V} / \mathrm{cm}$ field amplitude. Keeping in mind that $\mathrm{D}_{2}$ symmetry prohibits linear Stark effect we first attributed this to quadratic Stark effect. But actually when we applied a second pulse of the same duration and amplitude but opposite polarity the echo was revived with roughly the same amplitude. The revival also took place if the second Stark pulse had the same sign but was sent between the $\pi$-pulse and the echo.

"Corresponding author: ravil@kazanqc.org 
During the experiments we saw that the effect is present in 0 magnetic field as well as in 60 $\mathrm{mT}$ field oriented along the [001] crystallographic axis. These two revival conditions indicate that the observed Stark effect is linear despite the fact that it shouldn't be observed in the $\mathrm{D}_{2}$ site symmetry. This combination of suppression and revival of the echo may allow one to realize an addressing photon echo quantum memory protocol [2].

We attribute the linear Stark effect to the disturbances of the crystal field around the Tm ion sites that break the $\mathrm{D}_{2}$ symmetry. That gives each ion a phase shift different from the other ions. We also saw that the effect's magnitude is proportional to the frequency shift between the laser pulses carrier frequency and the inhomogeneous line centre.

Using the observed effect we realized address readout from the optical memory cell. We retrieved desired light pulses using electric pulses with amplitude of $500 \mathrm{~V} / \mathrm{cm}$ and 5 us duration. Fig. 1 shows the effect of different combinations of electric pulses and their compensating counterparts on the optical memory writing and reading process. Using the corresponding electric pulses we can suppress any one of the pulses. Using the additional $\pi$ pulse we can also retrieve pulses in the reversed order.

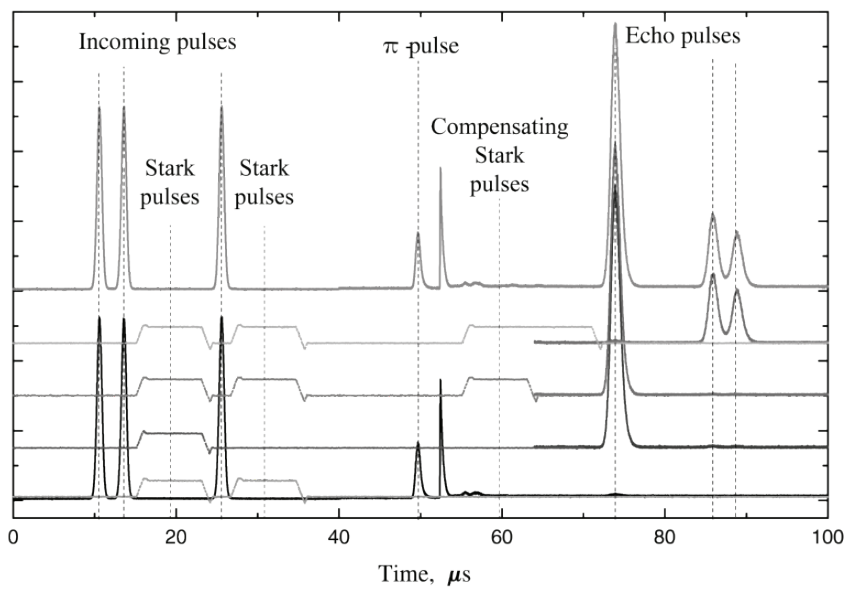

Fig. 1. Controlled retrieval of the photon echo signals using Stark pulses in $\mathrm{Tm}^{3+}: Y A G$ crystal. The darker solid curves show the incoming pulse sequences and echo pulses. The lighter solid lines show Stark pulses that suppress the echo and compensating pulses that revive desired echo pulses. There are always three incoming pulses - one pair and one single pulse. After them at 50 us time comes the rephasing $\pi$-pulse. Its tail can be seen at roughly 53 us at the lowest and the highest curves. The highest curve show the retrieval of all pulses and lowest curve - their full suppression. The second from the bottom curve shows the retrieval of just the single pulse. Above that - the suppression of all pulses and then revival of the single pulse using the additional compensating Stark pulse. Above that is the revival of both single pulse and the pair of pulses.

This research has been supported by the Government of Russian Federation, project no. 14.Z50.31.0040, Feb. 17, 2017.

\section{References}

[1] R.M. Macfarlane, J. Lumin. 125, 156 (2007)

[2] K.I. Gerasimov, M.M. Minnegaliev, S.A. Moiseev, R.V. Urmancheev, T. Chanelière and A. Louchet-Chauvet, Opt. Spectrosc. 123(2), 211 (2017) 